\section{Revise rules on conflicts of interest}

We contend that definitions of conflicts of interest (COI) in peer review need to be reassessed to reflect modern research practices. This could markedly increase the speed and quality of peer review.

For example, many potential reviewers are disqualified under current rules on co-authorship. However, research papers now have increasing numbers of co-authors and their interaction may be little more than episodic, with no genuine $\mathrm{COI}$ in practice. The judgement of an author who, say, contributed a data set to a paper is unlikely to be corrupted when reviewing a new paper from former co-authors.

In our editorial experience, co-authors typically have a sound understanding of each other's work and provide frank and constructive feedback. Using them as reviewers avoids settling for candidates who may be too far removed from the topic or not sufficiently senior in the field.

We suggest that only longrunning co-authorship should be counted as a COI in peer review. Other potential COIs should include supervisor-student relationships, shared institutional affiliations and collaborators working on the same project, with an expiry date if appropriate. Indrė Žliobaitė, Mikael Fortelius University of Helsinki, Finland. indre.zliobaite@helsinki.fi

\section{Preserve specimens for reproducibility}

The description of a new species without a preserved type specimen has always been permitted (T. Pape et al. Nature 537, 307; 2016) - but it should not become the norm. Original specimens allow testing of the hypotheses that underlie descriptions and so ensure reproducibility — an obligation and cornerstone of the scientific method.

It is taxonomic convention when describing a new species to deposit type specimens in a publicly accessible collection. This allows independent re-examination, reinterpretation and re-evaluation (Nature 535, 323-324; 2016). Although photographs can point to possible undescribed species and help to document biodiversity, they are open to misinterpretation (and also to manipulation).

Photographs alone should remain the exception, used only when specimens cannot be preserved for technical, legal or conservation reasons. Properly vouchered specimens are otherwise essential in biodiversity research, just as "laboratory notebooks and records must be available for independent review" in the experimental sciences (C. G. Begley et al. Nature 525, 25-27; 2015).

Frank T. Krell ${ }^{\star}$ Denver Museum of Nature \& Science, Colorado, USA.

frank.krell@dmns.org ${ }^{*}$ On behalf of 5 correspondents (see go.nature.com/2fiehxz for a full list).

\section{Open up research evaluation in China}

Strong academic opposition has led China's ministry of education to suspend a policy that would effectively control where Chinese researchers should publish their work. In my view, this scientifically disruptive intervention should never be reactivated.

The policy, launched by the ministry's Academic Degrees and Graduate Education Development Center (CDGDC) in April this year and repealed two weeks later, centred on an 'A-list' of top Chinese and international journals. The position of a journal in the list is determined by impact factor, years after the global movement away from its well-documented deficiencies as a tool for research assessment (see www.ascb.org/ dora). Entries also depend on inclusion in national databases, such as the Chinese Science Citation Database and the Chinese Social Sciences Citation Index. This could open the door to corruption as Chinese universities that publish journals vie for database entries.

China's Discipline Ranking (CDR) system intends to use the list to assess a university's performance by the number of its academics that publish in these journals. Rewards to scientists publishing in the 'top' journals might include payments and questionable promotions, for example, weakening the already distorted evaluation system and impeding the development of science in China.

I suggest that the CDGDC needs to be more serviceminded, recognizing that this contentious policy falls outside its authority. Making evaluation systems that are politically independent, non-profit and professional would help to break the CDGDC and CDR monopoly. Universities, too, should rethink the merit of political ranking lists.

Lihua Yang Beihang University, Beijing, China.

lihua.yang@buaa.edu.cn

\section{Australia too casual with protection law}

The Australian government has set a dangerous precedent in granting exemptions "in the national interest" from its 1999 Environment Protection and Biodiversity Conservation Act. This laxity adversely affects threatened species and disregards scientific advice.

Australia's former environment minister Greg Hunt granted the latest exemption. It permits habitat clearance in Batemans Bay, New South Wales, until the end of this year and dispersal by "non-lethal means" of camps of the threatened grey-headed flying fox (Pteropus poliocephalus). This species is crucial for native forest regeneration. Forced dispersals using loud noise, smoke and lights "often lead to flying fox stress, injuries, or fatalities" (see go.nature.com/2f9azyl) and have proved costly and inefficient (see B. J. Roberts et al. PLoS ONE 7, e42532; 2012).

Hunt also signed an exemption in 2014 to permit a shark cull in Western Australia (see go.nature. com/2f4mrdy), which included the protected great white shark (Carcharodon carcharias). Then there is the ongoing destruction of endangered forests in New South Wales, habitats for many threatened species.

In our view, Australia's federal and state governments should be more protective of biodiversity. Christian Vincenot Kyoto University, Japan.

Sophie Petit University of South Australia, Adelaide.

vincenot@i.kyoto-u.ac.jp

\section{Renewables from the bottom up}

Alan Bernstein and colleagues recommend a global grandchallenge strategy for prioritizing clean-energy research (Nature

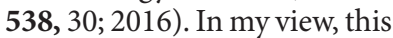
'cockpit' approach - relying on top-down steering to seek out global solutions - risks galvanizing research along a fixed path.

A diverse set of research initiatives implemented across different scales could be a better approach. That would deliver ways of using energy sustainably under different social and cultural circumstances. The long-term questions for energy research are as much about the evolution of energy use as the revolution in its production. Jari Lyytimäki Finnish Environment Institute, Helsinki, Finland. jari.lyytimaki@ymparisto.fi 
CORRECTION

Lihua Yang's Correspondence (Nature 539,

$168 ; 2016$ ) should have referred to possible

rewards to scientists publishing in 'top'

international journals, not just in Chinese journals. 